Check for updates

Cite this: RSC Adv., 2018, 8, 23623

\title{
Biocompatible surface modification of nano-scale zeolitic imidazolate frameworks for enhanced drug delivery $\dagger$
}

\begin{abstract}
Yuqing Li, Yongtai Zheng, (D) * Xinyi Lai, Yuehuan Chu and Yongming Chen (DD *
Recently, nanoscale metal organic frameworks have attracted considerable attention as a novel drug delivery system platform owing to their highly efficient drug loading and delivery capacities. However, their low dispersity in aqueous media, poor biocompatibility, and non-active targeting ability seriously limit their further clinical application. To address these issues, a hyaluronic acid (HA)-coated ZIF- 8 nanocomposite (CCM(aZIF-8/HA) was successfully developed for use in anti-cancer treatment through efficient curcumin (CCM) delivery. The resultant CCMaZIF-8/HA showed a long-term $\mathrm{pH}$-dependent controlled drug release based on the core-shell structure of the encapsulation by HA. Moreover, compared to bare CCMaZIF-8, the obtained ternary assembly showed enhanced dispersity in PBS, promoted cellular uptake, and greater growth inhibition against HeLa cells. Thus, CCM@ZIF-8/HA can not only serve as an ideal drug carrier, but it can also provide a general surface modification strategy to promote the performance of metal organic frameworks for efficient drug delivery.
\end{abstract}

Received 26th April 2018

Accepted 19th June 2018

DOI: $10.1039 / \mathrm{c} 8 \mathrm{ra03616k}$

rsc.li/rsc-advances

application. For example, the hydrophobicity of NMOFs leads

\section{Introduction}

Drug delivery systems (DDSs) are regarded as one branch of nanomedicine and promise targeted delivery and controlled release of drugs with minimal toxic effects compared to traditional chemotherapy. ${ }^{1-3}$ Over the past few decades, a rapid development of DDSs from organic carriers (e.g. liposomes, micelles and polymeric particles) to inorganic materials (e.g. mesoporous silica nanoparticles, quantum dots and metal oxides) has been witnessed. ${ }^{4-6}$ Very recently, nanoscale metal organic frameworks (NMOFs), a type of porous crystalline structure periodically assembled from metal ions and organic ligands, have attracted considerable attention as a novel DDS platform owing to their various compositions, multiple shapes and sizes, and tunable pores with large surface area. ${ }^{7-9}$ Among them, zeolite imidazolate framework-8 (ZIF-8) is considered to be one of the best candidates and has been continuously studied for use in DDSs due to several advantages: (i) compositions of $\mathrm{Zn}^{2+}$ and 2-methylimidazole are bio-friendly, (ii) the ease of its preparation at the nanoscale, (iii) its considerably high drug loading, and (iv) its biodegradability at $\mathrm{pH}<6 . .^{10-12}$

Despite their advantages, there are still some limitations remaining for NMOFs in terms of their further clinical

School of Materials Science and Engineering, Center for Functional Biomaterials, MOE Key Laboratory of Polymeric Composite and Functional Materials (PCFM), Sun Yat-sen University, Guangzhou 510275, China. E-mail: zhengyt@hotmail.com; chenym35@ mail.sysu.edu.cn

$\dagger$ Electronic supplementary information (ESI) available. See DOI: $10.1039 / \mathrm{c} 8 \mathrm{ra} 03616 \mathrm{k}$ them to agglomerate under physiological conditions, which inhibits the passive targeting of the so-called enhanced permeability and retention (EPR) effect. In addition, their poorly biocompatible surface means they are quickly removed by the reticuloendothelial system (RES) during blood circulation. ${ }^{13,14}$ Moreover, general NMOFs have no active targeting ability themselves, which may result in less accumulation at tumor sites with insufficient drug delivery. To overcome these limitations, several attempts have been made in terms of postsynthetic modifications or complexation. ${ }^{15-18}$ For example, Lázaro et al. modified UiO-66 with PEG for enhanced stability, ${ }^{19}$ Illes et al. coated MIL-88A with exosome for good biocompatibility, ${ }^{20}$ and Li et al. conjugated folic acid onto the Mi-UiO-68 for active targeting. ${ }^{21}$

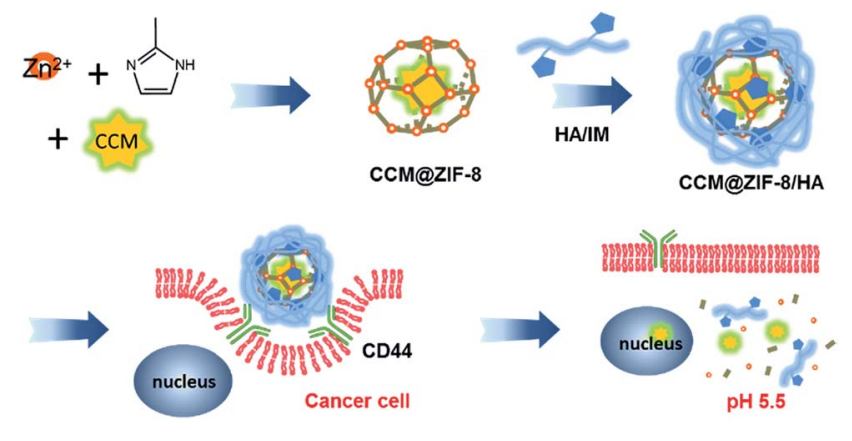

Scheme 1 Schematic representation of the preparation of the CCMaZIF-8/HA nanocomposite for targeted drug delivery in cancer cells. 

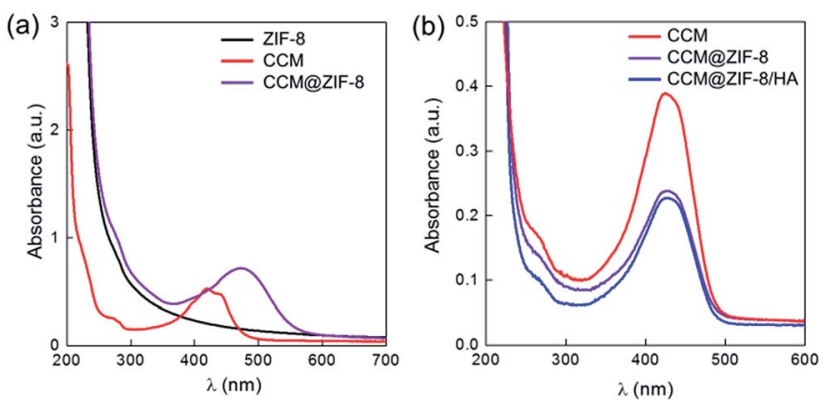

Fig. 1 UV-Vis absorption spectra of (a) ZIF-8, CCM, and CCMaZIF-8 and (b) CCM, CCMaZIF-8, and CCMaZIF-8/HA after digestion under acidic conditions.

In this study, we present a general approach for harnessing the surface of ZIF-8 for efficient curcumin (CCM) delivery. CCM is a natural phytochemical compound possessing potential as an anticancer drug, ${ }^{22}$ which can be further included in porous ZIF-8 (CCM@ZIF-8). ${ }^{23,24}$ Hyaluronic acid (HA) is a hydrophilic biopolymer that can actively bind to the CD44 receptor that is overexpressed by many growing tumor cells. Through complexing HA onto the surface of CCM@ZIF-8 via a coordinative interaction (CCM@ZIF-8/HA), CCM@ZIF-8/HA gains enhanced biocompatibility and additional active targeting ability. Consequently, the complexed nanoparticle is able to reach tumor sites and release the drug through the detachment of HA and the degradation of ZIF-8 under the influence of the low $\mathrm{pH}$ at the cancer tissue (Scheme 1). The strategy of such surface engineering can address the problems remaining for NMOFs in DDS applications, and thus has great potential in the clinical application of NMOFs for anti-cancer treatment.

\section{Results and discussion}

\section{Preparation and characterization of the CCM@ZIF-8/HA nanocomposite}

First, CCM@ZIF-8 was prepared via a one-step method from a methanol/water solution according to a previous report. ${ }^{24}$ The transmission electron microscopy (TEM) images showed nanoscaled particles with a uniform size of around $100 \mathrm{~nm}$ (Fig. S1 $\dagger$ ), and the hydrated radius was confirmed to be $73.5 \mathrm{~nm}$ using a nanoparticle size analyzer (Fig. S2 $\dagger$ ). The characteristic UV absorption for free CCM is around $420 \mathrm{~nm}$, but this is red shifted $58 \mathrm{~nm}$ to $478 \mathrm{~nm}$ for CCM@ZIF-8, suggesting that CCM has been successfully encapsulated into the channels of ZIF-8 (Fig. 1a). Moreover, imidazole (IM) substituted HA (HA/IM) was synthesized by conjugating histamine onto the HA backbone (Scheme S1 $\dagger$ ). The ${ }^{1} \mathrm{H}$ NMR measurements indicate that the grafting efficiency of IM is around $16 \%$ (Fig. S3 $\dagger$ ). Additionally, to examine the versatility for decorating polymers onto CCM@ZIF-8, an IM-pendent block copolymer of poly(ethylene glycol)- $b$-poly(histamine aspartate-co-benzyl aspartate) (mPEGPAsp/IM) synthesized from poly(ethylene glycol)- $b$-poly( $\beta$ benzyl L-aspartate) (mPEG-PBLA) was also prepared as a model polymer, where the substitution ratio for IM was around $17 \%$ (Fig. S4 $\dagger$ ). The final nanocomposite of CCM@ZIF-8/HA (or

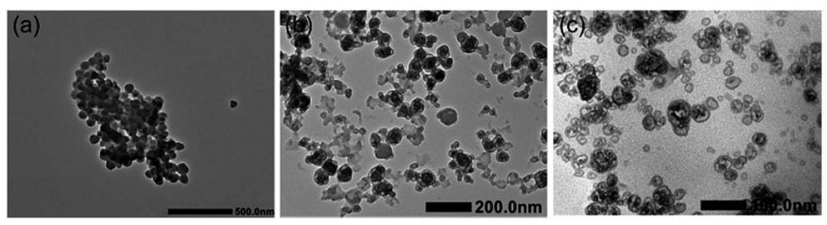

Fig. 2 TEM images for (a) CCMaZIF-8, (b) CCM@ZIF-8/mPEG-PAsp/ IM, and (c) CCMaZIF-8/HA prepared from dispersing in PBS.
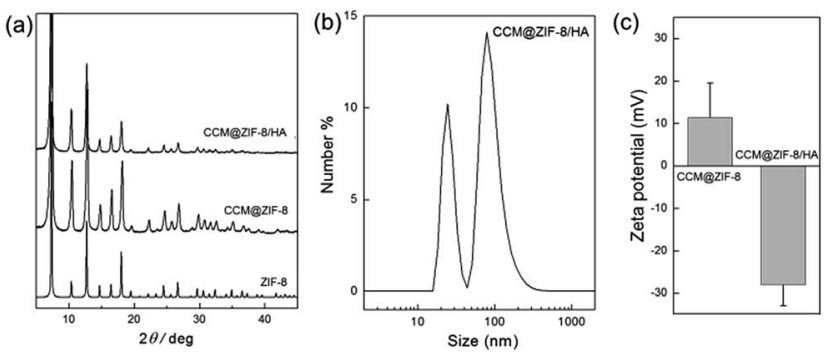

Fig. 3 (a) PXRD spectra of ZIF-8, CCMaZIF-8, and CCMaZIF-8/HA. (b) Hydrated radius determination for CCMaZIF-8/HA. (c) Zeta potential measurements for CCM@ZIF-8 and CCM@ZIF-8/HA.

CCM@ZIF-8/mPEG-PAsp/IM) was obtained by mixing a methanol solution of CCM@ZIF-8 and an aqueous solution of HA/IM (or mPEG-PAsp/IM) at room temperature and then heating at $60{ }^{\circ} \mathrm{C}$ for $1 \mathrm{~h}$. The drug loading capacity was evaluated using the standards of absorption intensities of CCM after digesting the particles of CCM@ZIF-8/HA under acidic conditions (Fig. S5 $\dagger$ ). The capacity of the encapsulated CCM was determined to be $4.24 \%$ for CCM@ZIF-8/HA, which is slightly lower than the value for CCM@ZIF-8 (4.50\%), indicating a limited loss of CCM during the surface modification process with HA (Fig. 1b). The morphologies of CCM@ZIF-8, CCM@ZIF-8/mPEG-PAsp/IM and CCM@ZIF-8/HA were characterized via TEM experiments (Fig. 2). The samples were prepared from the dispersion of each nanocomposite particle in phosphate-buffered saline (PBS) solution ( $\mathrm{pH}$ 7.4). It was found that the CCM@ZIF-8 particles significantly aggregated in PBS (Fig. 2a), which is quite distinct from the observation of their uniform dispersion in methanol (Fig. S1 $\dagger$ ). This should be ascribed to the hydrophobicity of the surface of CCM@ZIF-8, which leads them to being agglomerated in PBS. After being complexed with HA or mPEG-PAsp/IM, the nanocomposite particles exhibited a core-shell structure with obviously enhanced dispersity (Fig. 2b and c). The success of the surface coating is supposed to come from the coordination between the zinc ion of CCM@ZIF-8 and the IM residues of HA (or mPEG-PAsp/IM). ${ }^{25}$ Hence, the surface engineering strategy for CCM@ZIF-8 using hydrophilic polymers via coordinative interaction should be a versatile strategy. Interestingly, although CCM@ZIF-8/HA particles have been successfully obtained by coating with HA, some hollow structures of a smaller size were also observed (Fig. 2c). Indeed, HA alone only forms amorphous fibrous structures in PBS instead of hollow spheres (Fig. S6 $\dagger$ ). These hollow spheres are assumed to be from the degradation of parts of ZIF-8 inside CCM@ZIF-8/HA due to the 

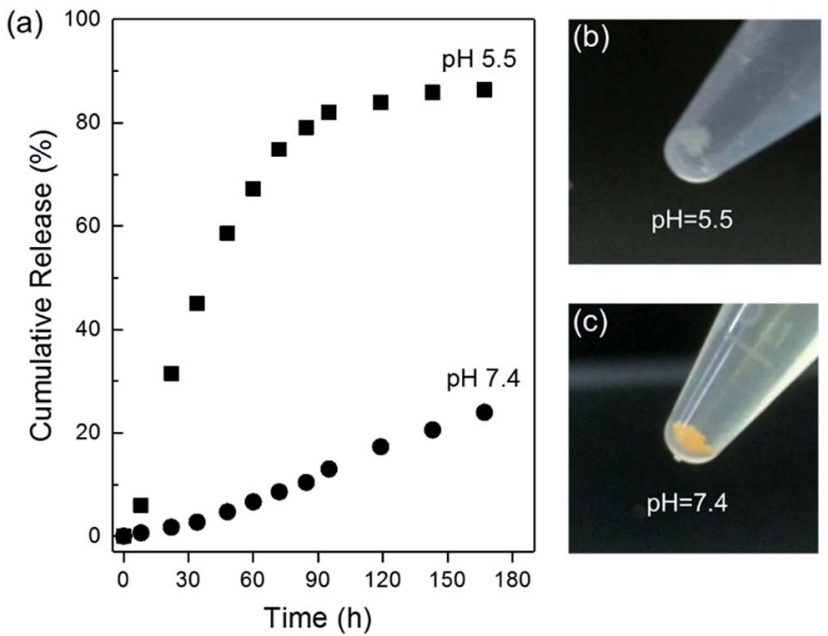

Fig. 4 (a) In vitro CCM release profiles for CCMaZIF-8/HA at $\mathrm{pH} 5.5$ and 7.4. Pictures of CCMaZIF-8/HA after one week at pH 5.5 (b) and 7.4 (c).

low stability of Zn-based MOFs at $60{ }^{\circ} \mathrm{C}$ with stirring during preparation. Also, the participation of the carboxylate groups of HA in the metal coordination accelerated the degradation of ZIF-8.

As seen in the powder X-ray diffraction (PXRD) patterns, the crystalline structure of ZIF-8 was retained completely after loading CCM (CCM@ZIF-8) and even after being complexed with HA (CCM@ZIF-8/HA) (Fig. 3a). There are two peaks assigned to the hollow and core-shell CCM@ZIF-8/HA (Fig. 3b). The hydrated radius of the core-shell CCM@ZIF-8/HA is around $159.2 \mathrm{~nm}$ with a PDI of 0.212 . These results are in accord with the observations from the TEM images. The zeta potential measurements indicate that the value for the initial surface of ZIF-8 is $+11.4 \mathrm{mV}$, but that this changes to $-28.1 \mathrm{mV}$ after it is covered by HA/IM (Fig. 3c). Moreover, the existence of each composition for CCM@ZIF-8/HA was determined using ${ }^{1} \mathrm{H}$ NMR measurements (Fig. S7†). These observations demonstrated the successful preparation of the surface modified CCM@ZIF-8/HA nanocomposite.

\section{pH-dependent in vitro drug release}

The in vitro drug release of CCM@ZIF-8/HA was monitored via the UV-Vis absorption of CCM in PBS with $0.1 \%$ Tween 80 at pH 5.5 and 7.4, which are close to the $\mathrm{pH}$ values of tumor tissues and normal tissues (Fig. 4a). At pH 7.4, the release of CCM is relatively slow and the final released amount is around $24 \%$ even after one week. In the whole process, no initial burst release was observed for CCM@ZIF-8/HA. This phenomenon is quite different from the behaviour of other conventional porous carriers, in which a burst release is frequently encountered for the absorbed drug molecules on the surface of the material. In our case, the high stability of the material is the main contributor to the protection of the HA/IM shell against the burst release and leakage of CCM in ZIF-8. In contrast, a biphasic release profile was found in acidic media at $\mathrm{pH}$ 5.5. The fast
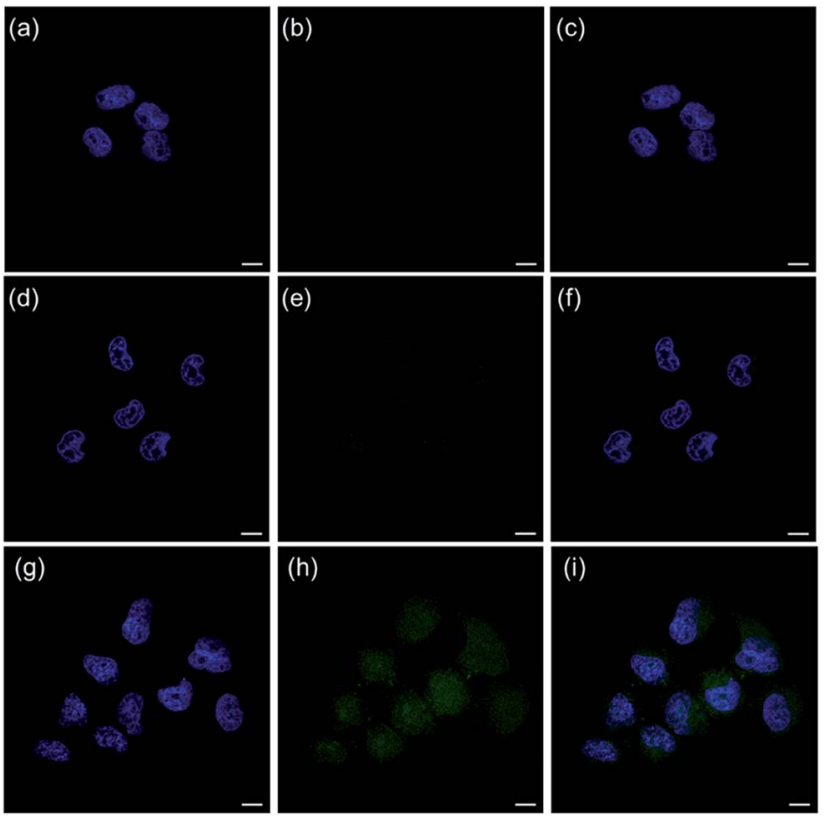

Fig. 5 CLSM images of HeLa cells incubated with free CCM $(a-c)$, CCMaZIF-8 $(d-f)$, and CCMaZIF-8/HA $(g-i)$, respectively. The cell nuclei were stained using DAPI (blue channel) and CCM (green channel). Scale bar: $25 \mu \mathrm{m}$.

release of CCM reached over $80 \%$ in the initial step of 4 days, and became saturated in the last 3 days. At a lower $\mathrm{pH}$, the protonated IM residues of HA/IM are no longer complexed with $\mathrm{Zn}^{2+}$, which leads to the detachment of the HA layer from the surface of CCM@ZIF- $8 .^{25}$ Thus the degradation of the resultant bare CCM@ZIF-8 was accelerated under acidic conditions with fast release of CCM. After incubation for one week, it became a pale floc at $\mathrm{pH} 5.5$ but still remained a fine yellow solid at $\mathrm{pH}$ 7.4 (Fig. 4b and c). Hence, CCM@ZIF-8/HA exhibits an excellent $\mathrm{pH}$ response for controlled drug release.

\section{Cellular internalization and cytotoxicity studies}

The cellular internalization for free CCM, CCM@ZIF-8 and CCM@ZIF-8/HA was monitored using confocal laser scanning microscopy (CLSM) after incubation with HeLa cells (Fig. 5). The CLSM analysis was carried out without any imaging probe since CCM itself emits green fluorescence. After $0.5 \mathrm{~h}$, slightly stronger fluorescence emission was observed for CCM@ZIF-8 than for free CCM, which might be attributed to the endocytosis effect of the cells. It is obvious that CCM@ZIF-8/HA shows the strongest fluorescence signal, suggesting an enhanced intracellular and CCM release ability. This should be attributed to the active targeting ability of the external HA layer, which further promotes the biocompatibility and favors cellular uptake.

In vitro cell viabilities were determined via MTT assay to evaluate the bio-toxicity for pure CCM, ZIF-8/HA, CCM@ZIF-8, and CCM@ZIF-8/HA, separately (Fig. 6). After incubation with HeLa cells in DMEM for $48 \mathrm{~h}$, the low toxicity of ZIF-8/HA confirmed that the cell viability was almost $65 \%$ even at 


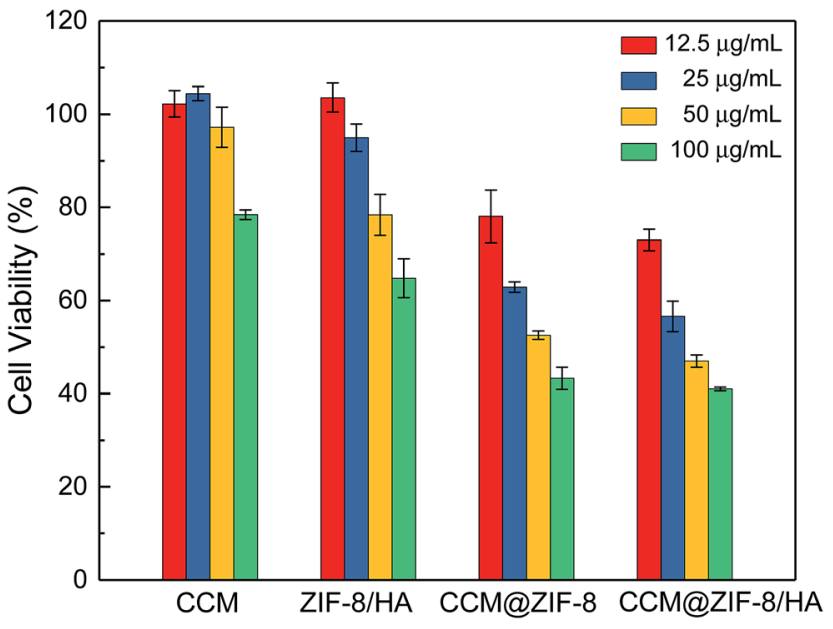

Fig. 6 Viabilities for HeLa cells in the presence of various concentrations of CCM, ZIF-8/HA, CCMaZIF-8, and CCMaZIF-8/HA.

a high concentration of $100 \mu \mathrm{g} \mathrm{mL}^{-1}$. This value is relatively high compared to the previous reports on bare ZIF-8, in which the cell viability is less than $40 \%$ at the same concentration, suggesting an improved biocompatibility for ZIF-8 after coating with HA. ${ }^{21}$ Compared to pure CCM, CCM@ZIF-8 showed a much enhanced cell growth inhibition, which originates from the lower degradation rate of CCM and the improved endocytosis for the CCM@ZIF-8 nanoparticles. After complexing with HA, CCM@ZIF-8/HA induced significant cell death despite the less cytotoxic HA coverage. This should be absolutely ascribed to the more efficient cellular uptake that comes from the active targeting ability of HA. Also, in contrast to ZIF-8/HA, the significant reduction in cell viability demonstrated the efficient intracellular drug release ability of CCM@ZIF-8/HA. Therefore, CCM@ZIF-8/HA has potential for application in cancer treatment with higher biocompatibility and more efficient tumor suppression.

\section{Experimental}

\section{Measurements}

The ${ }^{1} \mathrm{H}$ NMR spectra were measured on a Bruker AVANCE III $400 \mathrm{MHz}$ spectrometer using DMSO- $d_{6}$ or $\mathrm{D}_{2} \mathrm{O}$ as the solvent. Powder X-ray diffraction (PXRD) patterns were recorded using a Rigaku D/MAX 2200V PC diffractometer with $\mathrm{Cu} \mathrm{K}_{\alpha}$ radiation. UV-Vis absorption spectra were measured using a Thermo scientific evolution 201 at room temperature. Particle size distribution and zeta potential were determined using the Malvern Zeta Sizer Nano series with well-dispersed samples $\left(0.5 \mathrm{mg} \mathrm{mL}^{-1}\right)$ and each result was averaged from three measurements. Transmission electron microscopy (TEM) was performed on a JEM 1400 plus at $120 \mathrm{kV}$. The samples were prepared by drying $50 \mu \mathrm{L}$ solutions on a carboncoated copper grid at room temperature. Confocal laser scanning microscopy (CLSM) was carried out under a Carl Zeiss LSM 710 microscope. The MTT assay was tested using a Multiskan FC microreader.

\section{Materials}

Zinc nitrate hexahydrate $\left(\mathrm{Zn}\left(\mathrm{NO}_{3}\right)_{2} \cdot 6 \mathrm{H}_{2} \mathrm{O}\right)$, histamine dihydrochloride, and 1-(3-dimethylaminopropyl)-3-ethylcarbodiimide hydrochloride (EDC) were purchased from Adamas Reagent, Ltd. 2-Methylimidazole (MI), curcumin (CCM), and $N$-hydroxysuccinimide were purchased from Acros Organics. Hyaluronic acid (HA, MW $\sim 5.4 \mathrm{~K}$ ) was obtained from Freda BioChem Co., Ltd. Methanol and ethanol were obtained from the Guangzhou Chemical Reagent Factory. All chemicals were analytical reagent grade and were used without further purification. 3-(4,5dimethylthialzol-2-yl)-2,5-diphenyltetrazolium bromide (MTT) was purchased from Sigma-Aldrich Co., Ltd. Dulbecco's modified eagle medium (DMEM, Giboc), fetal bovine serum (FBS), phosphate buffered solution (PBS) and 4',6-diamidino-2phenylindole (DAPI) was purchased from Thermo Fisher Scientific. Centrifugal filter units (Millipore, Amicon Ultra-4, Ultracel-100k) were obtained from Merck Millipore. Poly(ethylene glycol)-b-poly( $\beta$-benzyl L-aspartate) (mPEG-PBLA) was kindly supplied by Prof. Shuai's group. ${ }^{26}$

\section{Preparation of CCM-loaded ZIF-8 (CCM@ZIF-8)}

CCM@ZIF-8 was obtained according to the previous report. ${ }^{24}$ To a stirring solution of CCM $(5 \mathrm{mg}, 0.01 \mathrm{mmol})$ and 2-MI (330 mg, $4.02 \mathrm{mmol})$ in methanol $(10 \mathrm{~mL}), \mathrm{Zn}\left(\mathrm{NO}_{3}\right)_{2} \cdot 6 \mathrm{H}_{2} \mathrm{O}(150 \mathrm{mg}$, $0.504 \mathrm{mmol})$ in water $(5 \mathrm{~mL})$ was poured and allowed to react for $1 \mathrm{~min}$ at room temperature. The resultant precipitate was collected via centrifugation (10k rpm, $15 \mathrm{~min}$ ) and then washed with fresh methanol three times. An orange solid was obtained after drying under vacuum at room temperature. Yield: $30 \mathrm{mg}$, $26 \%$. The content of CCM in ZIF-8 was determined using a UVVis spectrophotometer at $\lambda=428 \mathrm{~nm}$ after decomposing CCM@ZIF-8 in $\mathrm{HCl}$ aqueous solution. The final drug loading capability of CCM was determined to be $4.5 \%$.

\section{Synthesis of IM-pendent HA (HA/IM)}

HA/IM was prepared according to a previous report. ${ }^{27}$ Determined amounts of EDC $(15 \mathrm{mg}, 0.08 \mathrm{mmol})$ and NHS $(9 \mathrm{mg}$, $0.08 \mathrm{mmol})$ were added into a $\mathrm{HA}$ aqueous solution $(10 \mathrm{mg}$ $\mathrm{mL}^{-1}$ ) under stirring. After $30 \mathrm{~min}$, histamine dihydrochloride ( $8 \mathrm{mg}, 0.04 \mathrm{mmol}$ ) was added. The reaction solution was further stirred for $24 \mathrm{~h}$ at room temperature. The resultant mixture was dialyzed against excess water for 3 days. A white powder was finally obtained through freeze-drying. Yield: $270 \mathrm{mg}$.

\section{Synthesis of modified poly(ethylene glycol)-b-poly(histamine aspartate-co-benzyl-aspartate) (mPEG-PAsp/IM)}

mPEG-PAsp/IM was obtained according to the previous report. ${ }^{26}$ Histamine dihydrochloride ( $139 \mathrm{mg}, 0.757 \mathrm{mmol}$ ) and mPEGPBLA (250 mg, $0.0473 \mathrm{mmol}$ ) were added to $2 \mathrm{~mL}$ anhydrous DMSO in a reaction vial under argon. Then $0.21 \mathrm{~mL}(1.5 \mathrm{~mol})$ TEA was added. The reaction was conducted for $7 \mathrm{~h}$, and then the solution was dialyzed (MWCO: $1.0 \mathrm{kDa}$ ) against methanol for $72 \mathrm{~h}$ and dried via rotary evaporation to obtain mPEG-PAsp/ IM. Yield: $191 \mathrm{mg}$. 


\section{Preparation of CCM@ZIF-8/HA}

To a solution of IM-HA (15 mg, $2.7 \mathrm{~mol})$ in water $(1.5 \mathrm{~mL})$, CCM@ZIF-8 (15 mg, $0.66 \mathrm{mmol})$ in methanol $(1.5 \mathrm{~mL})$ was poured under stirring at room temperature, and the resultant solution was mixed at $60{ }^{\circ} \mathrm{C}$ for $1 \mathrm{~h}$. After removing the unreacted reagents in solution via centrifugation (10k rpm), the solid residue was resuspended in water and collected at a lower speed $(1.5 \mathrm{k} \mathrm{rpm})$ to avoid aggregation. The final product was obtained as an orange powder through freeze-drying. Yield: $10 \mathrm{mg}, 30 \%$.

\section{Preparation of CCM@ZIF-8/mPEG-PAsp/IM}

To a solution of mPEG-PAsp/IM (15 mg, $8.5 \mathrm{mmol})$ in methanol (1.5 mL), CCM@ZIF-8 (15 mg, $0.66 \mathrm{mmol})$ in methanol $(1.5 \mathrm{~mL})$ was poured under stirring at room temperature, and the resultant solution was mixed at $60^{\circ} \mathrm{C}$ for $1 \mathrm{~h}$. After removing the unreacted reagents in solution via centrifugation (10k rpm), the solid residue was resuspended in water and collected at a lower speed $(1.5 \mathrm{k} \mathrm{rpm})$ to avoid aggregation. The final product was obtained as an orange powder through freeze-drying. Yield: $10 \mathrm{mg}, 30 \%$.

\section{In vitro drug release}

The suspension of CCM@ZIF-8/HA (2.4 mg) in 3 mL PBS (pH 5.0 or 7.4) containing Tween-80 (1.0 wt\%) in centrifugal filter units was incubated at $37{ }^{\circ} \mathrm{C}$ under oscillation. The amount of released CCM was collected and determined using a UV-Vis spectrophotometer at $428 \mathrm{~nm}$ at certain time intervals.

\section{Confocal laser scanning microscopy (CLSM)}

A HeLa cell solution $(1 \mathrm{~mL})$ in DMEM media containing $10 \%$ FBS was placed into a 6-well culture plate (an amicrobic cover slip was placed in each well) at a density of $1 \times 105$ cells per well, and was allowed to adhere overnight. Then, the cells were incubated with the samples for $0.5 \mathrm{~h}$ at $37{ }^{\circ} \mathrm{C}$. After removing the suspensions, the cells were washed with PBS $(1 \mathrm{~mL} \times 2)$, fixed with $4 \%$ formaldehyde $(1 \mathrm{~mL}$ ) for $10 \mathrm{~min}$, and then washed with PBS once more. Finally, the slides were mounted and observed under a microscope (Carl Zeiss LSM 710) after staining the nuclei with DAPI.

\section{In vitro cytotoxicity experiments}

Cell toxicity was quantified using an MTT assay. HeLa cells were routinely cultured in DMEM media containing 10\% FBS, seeded into a 96-well plate at a density of $1 \times 104$ cells per well, and incubated at $37{ }^{\circ} \mathrm{C}$ in a humidified atmosphere with $5 \% \mathrm{CO}_{2}$ for $24 \mathrm{~h}$. Subsequently, the cells were incubated in various concentrations of free CCM, ZIF-8/HA, CCM@ZIF-8, and CCM@ZIF-8/HA for another $48 \mathrm{~h}$. The CCM was pre-dissolved in DMSO $\left(1 \mathrm{mg} \mathrm{mL}^{-1}\right)$ and diluted with PBS. After incubating the cells with MTT (15 mg mL ${ }^{-1}$ ) for $4 \mathrm{~h}$, the solvent was changed to DMSO for dissolving formazan. The amount of formazan was obtained by recording the absorbance at $\lambda=490 \mathrm{~nm}$ using a microreader (Multiskan FC).

\section{Conclusions}

We have developed a feasible method to prepare biopolymerdecorated ZIF-8 for promoted CCM delivery (CCM@ZIF-8/HA), which was well characterized using UV-Vis, TEM, PXRD and zeta potential. It showed a long-term $\mathrm{pH}$-dependent controlled drug release based on the core-shell structure of CCM@ZIF-8/ HA. It also exhibited enhanced biocompatibility and cellular uptake against HeLa cells with efficient anticancer ability. In view of the rapid development of NMOFs in DDSs, we believe that the strategy presented here could promote the performance of NMOF-based anti-cancer carriers with excellent aqueous dispersity, biocompatibility, and active targeting ability.

\section{Conflicts of interest}

There are no conflicts to declare.

\section{Acknowledgements}

This project was supported by the National Natural Science Foundation of China (No. 51503230), the Guangdong Innovative and Entrepreneurial Research Team Program (No. 2013S086), and the Natural Science Foundation of Guangdong Province (No. 2016A030310465, 2014A030312018).

\section{Notes and references}

1 J. A. Hubbell and A. Chilkoti, Science, 2012, 337, 303-305.

2 K. Bourzac, Nature, 2012, 491, 58-60.

3 J. Shi, A. R. Votruba, O. C. Farokhzad and R. Langer, Nano Lett., 2010, 10, 3223-3230.

4 T. Lammers, W. E. Hennink and G. Storm, Br. J. Cancer, 2008, 99, 392-397.

5 G. Chen, I. Roy, C. Yang and P. N. Prasad, Chem. Rev., 2016, 116, 2826-2885.

6 E.-K. Lim, T. Kim, S. Paik, S. Haam, Y.-M. Huh and K. Lee, Chem. Rev., 2015, 115, 327-394.

7 P. Horcajada, C. Serre, M. Vallet-Regí, M. Sebban, F. Taulelle and G. Férey, Angew. Chem., Int. Ed., 2006, 45, 5974-5978.

8 P. Horcajada, T. Chalati, C. Serre, B. Gillet, C. Sebrie, T. Baati, et al., Nat. Mater., 2010, 9, 172-178.

9 W. Cai, C.-C. Chu, G. Liu and Y.-X. J. Wáng, Small, 2015, 11, 4806-4822.

10 J. Zhuang, C.-H. Kuo, L.-Y. Chou, D.-Y. Liu, E. Weerapana and C.-K. Tsung, ACS Nano, 2014, 8, 2812-2819.

11 N. Liédana, A. Galve, C. Rubio, C. Téllez and J. Coronas, ACS Appl. Mater. Interfaces, 2012, 4, 5016-5021.

12 C. Adhikari, A. Das and A. Chakraborty, Mol. Pharmaceutics, 2015, 12, 3158-3166.

13 L. Zhang, Y. Li and J. C. Yu, J. Mater. Chem. B, 2014, 2, 452470.

14 R. A. Petros and J. M. DeSimone, Nat. Rev. Drug Discovery, 2010, 9, 615-627.

15 M.-X. Wu and Y.-W. Yang, Adv. Mater., 2017, 1606134.

16 E. Bellido, T. Hidalgo, M. V. Lozano, M. Guillevic, R. SimónVázquez, et al., Adv. Healthcare Mater., 2015, 4, 1246-1257. 
17 V. Agostoni, P. Horcajada, M. Noiray, M. Malanga, A. Aykaç, et al., Sci. Rep., 2015, 5, 7925.

18 T. Hidalgo, M. Giménez-Marqués, E. Bellido, J. Avila, M. C. Asensio, et al., Sci. Rep., 2017, 7, 43099.

19 I. A. Lázaro, S. Haddad, S. Sacca, C. Orellana-Tavra, D. Fairen-Jimenez and R. S. Forgan, Chem, 2017, 2, 561-578.

20 B. Illes, P. Hirschle, S. Barnert, V. Cauda, S. Wuttke and H. Engelke, Chem. Mater., 2017, 29, 8042-8046.

21 Y.-A. Li, X.-D. Zhao, H.-P. Yin, G.-J. Chen, S. Yanga and Y.-B. Dong, Chem. Commun., 2016, 52, 14113-14116.

22 P. Anand, A. B. Kunnumakkara, R. A. Newman and B. B. Aggarwal, Mol. Pharm., 2007, 4, 807-818.
23 A. Tiwari, A. Singh, N. Garg and J. K. Randhawa, Sci. Rep., 2017, 7, 12598.

24 M. Zheng, S. Liu, X. Guan and Z. Xie, ACS Appl. Mater. Interfaces, 2015, 7, 22181-22187.

25 R. Röder, T. Preiß, P. Hirschle, B. Steinborn, A. Zimpel, M. Höhn, et al., J. Am. Chem. Soc., 2017, 139, 2359-2368.

26 L. Zhang, H. Xiao, J. Li, D. Cheng and X. Shuai, Nanoscale, 2016, 8, 12608-12617.

27 M. Nakanishi, J. Park, W. Jang, M. Oba and K. Kataoka, React. Funct. Polym., 2007, 67, 1361-1372. 